\title{
MIR370 Pre-miRNA
}

National Cancer Institute

\section{Source}

National Cancer Institute. MIR370 Pre-miRNA. NCI Thesaurus. Code C82824.

MIR370 is an oligoribonucleotide that is encoded by the human MIR370 gene and has a role in the regulation of gene expression. 